\title{
Electrical Stimulation of Broca's Area Enhances Implicit Learning of an Artificial Grammar
}

\author{
Meinou H. de Vries ${ }^{1,2 *}$, Andre C. R. Barth ${ }^{1 *}$, Sandra Maiworm ${ }^{1}$, \\ Stefan Knecht ${ }^{1,3}$, Pienie Zwitserlood ${ }^{1}$, and Agnes Flöel ${ }^{1,4}$
}

\begin{abstract}
Artificial grammar learning constitutes a well-established model for the acquisition of grammatical knowledge in a natural setting. Previous neuroimaging studies demonstrated that Broca's area (left BA 44/45) is similarly activated by natural syntactic processing and artificial grammar learning. The current study was conducted to investigate the causal relationship between Broca's area and learning of an artificial grammar by means of transcranial direct current stimulation (tDCS). Thirty-eight healthy subjects participated in a between-subject design, with either anodal tDCS (20 min, $1 \mathrm{~mA}$ ) or sham stimulation, over Broca's area during the acquisition of an artificial grammar. Performance during the acquisition phase, presented as a working memory task, was comparable between groups. In the subsequent classification task,
\end{abstract}

\section{INTRODUCTION}

How humans learn and communicate through language is an outstanding scientific challenge to understand (Petersson, Forkstam, \& Ingvar, 2004; see also Hauser, Chomsky, \& Fitch, 2002; Jackendoff, 2002; Chomsky, 2000). Extracting complex, rule-based information from the environment is an intrinsic feature for the acquisition of a language's grammar. Despite the lack of explicit knowledge about the underlying grammatical rules, native speakers are usually capable of producing errorless sentences of great complexity. This accomplishment is due to the implicit fashion in which grammatical rules of a language are learned in childhood.

Implicit learning has been the focus of investigation for many decades. Learning is implicit when we acquire new information without intending to do so, often in such a way that the acquired knowledge is difficult to verbally express. Explicit learning, such as learning how to solve a problem or learning a new concept, is an active process, typically hypothesis-driven, and thus, fully conscious (Cleeremans, Destrebecqz, \& Boyer, 1998). In order to investigate implicit learning in general, as well as the way in which we implicitly

\footnotetext{
${ }^{1}$ University of Münster, Germany, ${ }^{2}$ Max Planck Institute for Psycholinguistics, Nijmegen, The Netherlands, ${ }^{3}$ Neurocenter at SchönKlinik Hamburg-Eilbek, Germany, ${ }^{4}$ Universitätsmedizin Charite, Berlin, Germany

*The two first authors contributed equally to this work.
}

detecting syntactic violations, and specifically, those where no cues to superficial similarity were available, improved significantly after anodal tDCS, resulting in an overall better performance. A control experiment where 10 subjects received anodal tDCS over an area unrelated to artificial grammar learning further supported the specificity of these effects to Broca's area. We conclude that Broca's area is specifically involved in rule-based knowledge, and here, in an improved ability to detect syntactic violations. The results cannot be explained by better tDCS-induced working memory performance during the acquisition phase. This is the first study that demonstrates that tDCS may facilitate acquisition of grammatical knowledge, a finding of potential interest for rehabilitation of aphasia.

learn a language, Arthur Reber instantiated the so-called artificial grammar learning (AGL) paradigm (Reber, 1967). In the original task, subjects were asked to memorize a set of letter strings generated by a finite state grammar, schematically displayed in Figure 1. Subsequently, they were told that the previously presented strings followed a certain grammar; and they were asked to decide whether or not novel strings are grammatical. Typically, participants perform this task reliably above chance, despite the fact that they are unable to verbally describe the rules of the grammar. Crucially, this extraction of abstract information from the environment is intrinsic to natural language learning (Reber, 1967).

Several studies have investigated the neural correlates of implicit AGL. Broca's area has repeatedly been found to be involved in AGL paradigms, as demonstrated in fMRI experiments (e.g., Forkstam, Hagoort, Fernández, Ingvar, \& Petersson, 2006; Petersson et al., 2004), TMS experiments (Udden et al., 2008), and studies of white matter integrity, using MR-based diffusion tensor imaging (Flöel, De Vries, Scholz, Breitenstein, \& Johansen-Berg, 2009). More specifically, Broca's area has been implicated in rule-based knowledge, and especially in syntactic violation detection.

The first characteristic, that is, rule-based knowledge, has been demonstrated in several neuroimaging studies that investigated the role of Broca's area in AGL. Rulebased knowledge (i.e., discriminating items based on their structural resemblance) as opposed to chunk-based knowl- 


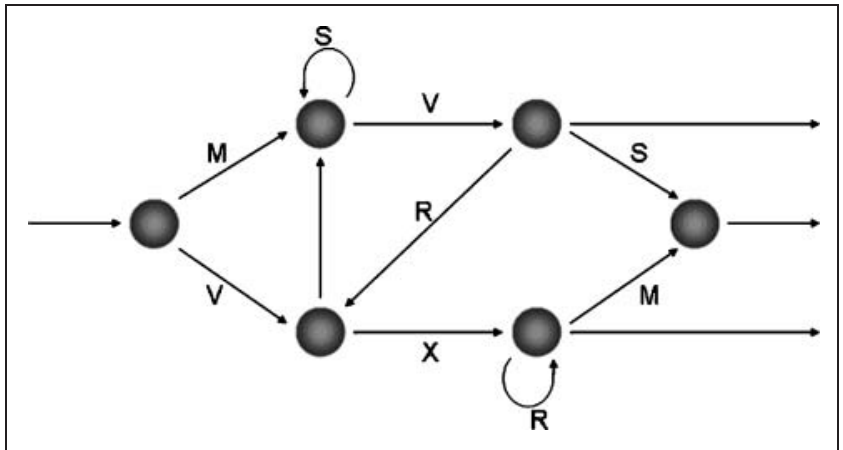

Figure 1. The grammar used for generating stimuli in the AGL paradigm by Reber (1967).

edge (i.e., discriminating items based on their superficial resemblance) was found to specifically engage Broca's area (Forkstam et al., 2006; Opitz \& Friederici, 2003, 2004).

The second characteristic, specific involvement of Broca's area in detecting syntactic violations, is a very robust finding in previous imaging studies. For instance, both Forkstam et al. (2006) and Petersson et al. (2004) found that Broca's area was specifically engaged in the processing of syntactic violations. Furthermore, this could also be replicated in a brain stimulation study: Udden et al. (2008) applied rTMS during the classification phase of an AGL task and, indeed, supported a causal role for Broca's area in syntactic violation detection.

However, these previous studies focused on the classification test, and not on the acquisition process. The crucial question of whether Broca's area is causally involved in the acquisition process of grammatical knowledge has not been answered so far.

In the current experiment, we electrically stimulated Broca's area during the acquisition of an artificial grammar, instead of focusing on the classification task, as done previously (cf. Udden et al., 2008). We used anodal transcranial direct current stimulation (tDCS), a technique that has been shown to increase cortical excitability (Purpura \& McMurtry, 1965), and NMDA receptor-dependent plasticity (Liebetanz, Nitsche, Tergau, \& Paulus, 2002). Moreover, tDCS has been shown to modulate motor learning (Reis et al., 2009; Nitsche, Schauenburg, et al., 2003), probabilistic classification learning (Kincses, Antal, Nitsche, Bartfai, \& Paulus, 2004), and learning of a novel lexicon (Flöel, Roesser, Michka, Knecht, \& Breitenstein, 2008). Our aim was to elucidate the causal role of Broca's area in the acquisition of an artificial grammar, specifically to determine its role in the two most prominent features of AGL: syntactic violation detection and rule-based learning, as opposed to chunk-based learning.

In order to do so, we used two sets of items with different relative chunk strength (i.e., frequency of bi- and trigrams in the test items relative to the training items; cf. Lieberman, Chang, Chiao, Bookheimer, \& Knowlton, 2004; Meulemans \& Van der Linden, 1997): One set contained strings that were highly familiar to the strings in the acquisition phase, evoking performance based on superficial similarity (chunkbased knowledge), the other set contained strings that had little superficial resemblance to the acquisition strings, evoking performance based on structural similarity (rule-based knowledge). This manipulation was independent of the grammaticality status of the strings, allowing us to analyze the effect of tDCS on both syntactic violation detection and rule-based knowledge in a factorial design (see Forkstam et al., 2006, and Udden et al., 2008, for a similar approach).

\section{METHODS}

\section{Subjects}

Forty-four participants were originally recruited into the study (19 women, mean age $=22.6$ years, $S D=2.1$ ) for a small financial reimbursement or study credits. All participants were right-handed and all were university students (mean education in years $=15.6, S D=1.5$ ). They had no history of chronic or acute neurologic, psychiatric, or medical disease; no family history of epilepsy; no current pregnancy; no cardiac pacemaker; no previous surgery involving implants to the head (cochlear implants, aneurysm clips, brain electrodes); and did not take acute or chronic medication or drugs.

Post hoc, 10 additional participants with demographic characteristics comparable to the other two groups ( 5 women; mean age $=23.7, S D=2.4$; mean education in years $=15.3, S D=1.34$ ) were recruited for a control experiment, in which we stimulated $\mathrm{Cz}$ (of the International 10-20 EEG System), an area that has not been implicated in AGL tasks.

\section{Cognitive Screening}

Prior to participating in the experiment, the participants were assessed with a neuropsychological test battery, including tests of general intellectual performance, working memory, and attention. Furthermore, blood pressure and the Positive and Negative Affective Schedule (PANAS; Watson, Clark, \& Tellegen, 1988) were assessed before and after the experimental session.

\section{Artificial Grammar Learning}

\section{General Experimental Outline}

The experiment consisted of an acquisition phase and a classification phase. In total, the experiment lasted about $40 \mathrm{~min}$ (see Figure 2 for the experimental timeline). The participants were randomly assigned to either the stimulation or sham group, resulting in 22 participants for each group.

\section{Stimuli}

The stimuli were generated from the finite state grammar as depicted in Figure 1. Examples of valid letter strings are MSSV, VXVRXRM, and MVRXRRM, derived by progressing 
Figure 2. Timeline of the experiment (top) and stimulation site (bottom). The stimulating electrode was placed over Broca's area (left BA 44/45) as delineated according to the International 10-20 EEG System.

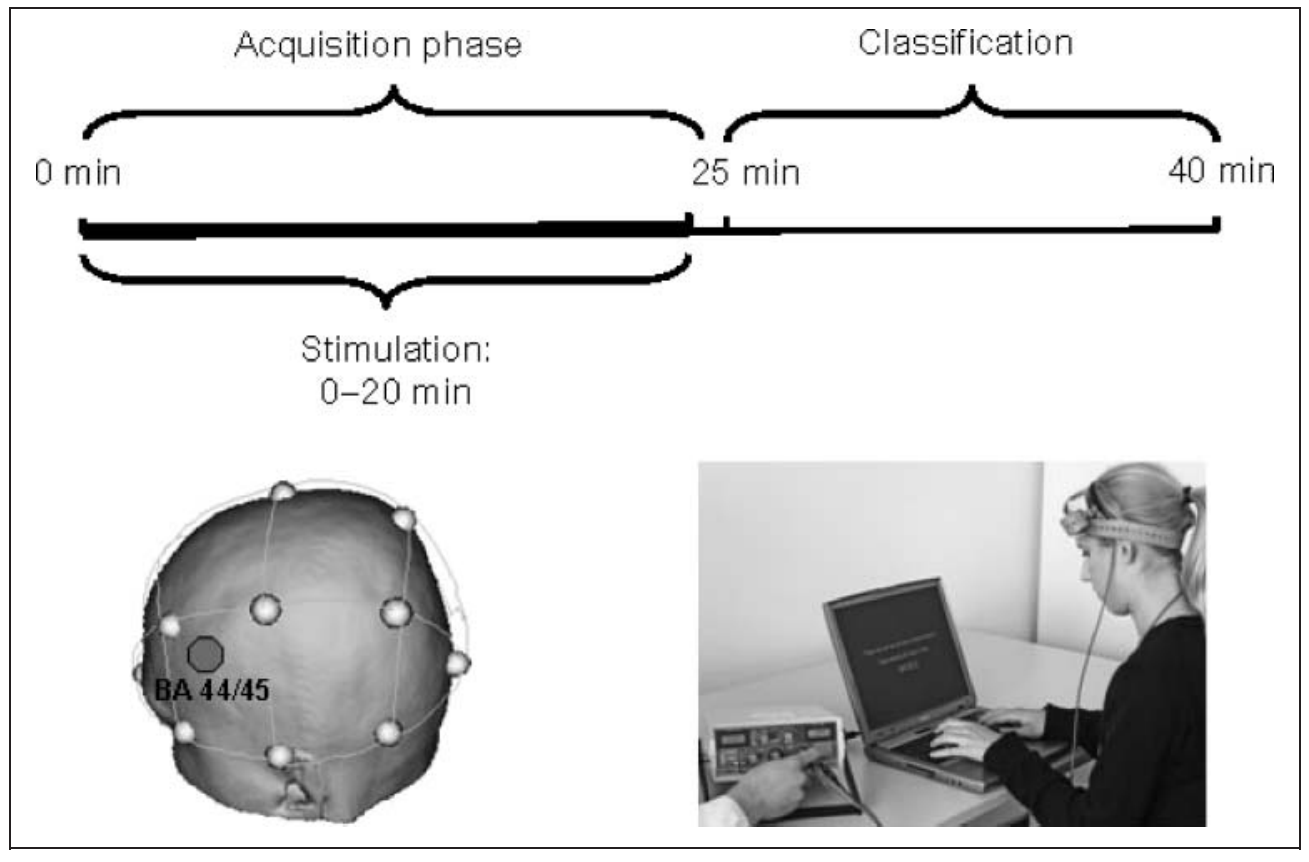

from node to node through valid transitions (the arrows), from start to end. We generated 200 grammatical strings from this grammar with a string length of 5-12 letters. One hundred strings were used for the acquisition phase, and 100 for the classification phase, of which 50 strings were nongrammatical. We calculated the chunk strength (i.e., the degree of superficial familiarity of a test string to a string in the acquisition phase). First, we counted the number of times a bi- or trigram appeared in the acquisition set. For instance, the chunk "VRX" appeared relatively often in the acquisition set and would therefore have a high chunk strength. The total chunk strength of a test string was then derived by averaging the chunk strength of all chunks in this particular test string (cf. Meulemans \& Van der Linden, 1997; Knowlton \& Squire, 1996). Two sets of items were created: one set containing 50 items with high, and one set containing 50 items with low chunk strength, relative to the acquisition set. From each item sample, 25 items were taken to create nongrammatical test strings, such that chunk strength was independent of a string's grammaticality status. The nongrammatical test strings were derived by a switch of letters in two nonterminal positions, while keeping the chunk strength relatively intact (the relative chunk strength for the grammatical items was 102, for nongrammatical items 97, for low chunk strength items 91, for high chunk strength items 108). All strings were presented in random order.

\section{Implicit Acquisition Task}

For the implicit acquisition task (with a duration of approximately $25 \mathrm{~min}$ ), the participants were told that this was a working memory task. They were instructed to closely pay attention to the string that was presented on a computer screen, and then to recall it as accurately as possible by typing the string on the key board. Timing was self-paced and participants were allowed to correct their input by using the back-space button. Each string was presented in its complete form for $5 \mathrm{sec}$ and was centrally placed on the computer screen. The experiment was programmed using the Presentation software (nbs.neuro-bs.com). Only grammatical exemplars were presented, and no performance feedback was provided.

\section{Classification Task}

Prior to the classification task, the participants were told that the strings they had been presented with during the acquisition task were all generated from a certain grammar. For the classification task (with a duration of approximately 15 minutes), they were instructed to classify novel strings as grammatical or nongrammatical by using the corresponding response buttons. They had to respond as accurately and as quickly as possible, relying on their immediate intuition. 100 strings were presented on the screen, one at a time. 50 were grammatical ( 25 high chunk strength, 25 low chunk strength), and 50 nongrammatical (25 high chunk strength, 25 low chunk strength).

\section{Transcranial Direct Current Stimulation}

tDCS was delivered by a battery-driven constant DC current stimulator (Schneider Electronic, Gleichen, Germany) through two electrodes. Constant current flow was controlled by an amperemeter. The stimulating electrode (to which the term anodal stimulation refers) was inserted in a $5 \times 7 \mathrm{~cm}$ saline-soaked synthetic sponge, and centered over left BA 44/45 (position delineated according to the International 10-20 EEG System; using the Münster T2T-converter to determine the position on the EEG 
system; http://wwwneuro03.uni-muenster.de/ger/t2tconv/; see Figure 2 above). The second electrode (reference, inserted in a $10 \mathrm{~cm} \times 10 \mathrm{~cm}$ saline-soaked synthetic sponge) was positioned over the contralateral supraorbital region. The increased size of the fronto-polar reference electrode renders stimulation over this cortex functionally inefficient without compromising the tDCS-generated effects under the active electrode (Nitsche et al., 2007). Anodal tDCS (current of $1 \mathrm{~mA}$ ) was delivered for 20 min during which the acquisition phase took place. For the participants who were assigned to the sham group, an ultra-short (<30 sec) stimulation at the start of the session was delivered. At the onset of each session (for both the anodal tDCS and sham group), the current was increased in a ramp-like fashion (Nitsche, Liebetanz, et al., 2003), eliciting a transient tingling sensation on the scalp that faded over seconds, consistent with previous reports (Nitsche, Schauenburg, et al., 2003). For both groups, currents were then turned off slowly over a few seconds (for the tDCS group after $20 \mathrm{~min}$ of stimulation, for the sham group after $30 \mathrm{sec}$ of stimulation), out of the field of view of the participants, a procedure that does not elicit perceptions (Hummel et al., 2005; Nitsche, Schauenburg, et al., 2003).

Implicit grammar learning started at the beginning of the stimulation and continued after the end of tDCS or sham stimulation (20 min) for another 5 min (see Figure 2).

\section{Statistical Analysis}

For the acquisition phase, we calculated the number of correct recalls for each participant, and subsequently examined if there were group differences by using Student's $t$ tests.

For the classification phase, we were interested in the two parameters described in the Introduction: syntactic violation detection and rule-based knowledge.

Previous work by Lieberman et al. (2004) demonstrated that for low chunk strength items, which are naturally less similar to training items, accurate discrimination of grammatical from nongrammatical items must rely on knowledge of the grammar rules. On the other hand, if there are available superficial similarities, or chunk strength cues, which is the case for high chunk strength items, accurate discrimination relies on chunk - rather than on rule-based knowledge. Separation of performance on low and high chunk strength items thus enabled us to investigate rulebased knowledge as opposed to chunk-based knowledge. Separation of performance on nongrammatical and grammatical items allowed us to specifically investigate if potential performance improvement was driven by syntactic violation detection as opposed to a better ability to identify grammatical items.

Syntactic violation detection is investigated through separating grammatical and nongrammatical items. We expected to find a lower false alarm rate for the tDCS compared to the sham group. Rule-based knowledge is investigated through separating low and high chunk strength items. We expected to find a better performance on the low chunk strength items for the tDCS compared to the sham group.

Therefore, we calculated $d^{\prime}$ values, using standard signal detection theory (Hochhaus, 1972), for both high and low chunk strength items samples. Not only does the $d^{\prime}$ statistic control for response bias, it also allowed us to directly compare our results with our previous study (Flöel et al., 2009), where we found that white matter integrity around Broca's area was correlated with the $d^{\prime}$ value for the low chunk strength items (i.e., rule-based knowledge).

Furthermore, we calculated the hit rates for the grammatical items (of both high and low chunk strength) and false alarm rates for the nongrammatical items (of both high and low chunk strength). This constituted the dependent variable "endorsement rate" (i.e., items perceived as grammatical), a common variable in the AGL literature to assess syntactic violation detection (Udden et al., 2008; Forkstam et al., 2006; Lieberman et al., 2004; Knowlton \& Squire, 1996).

\section{RESULTS}

Six participants originally recruited into the study had to be excluded due to extremely slow reaction times (>1000 msec, corresponding to >2 SDs of mean RT; $623 \mathrm{msec}, S D=193 \mathrm{msec}$ ), which may reflect to explicit problem solving rather than immediate and intuitionbased responding, as had been instructed (see Howard \& Howard, 2001, for a review). Thus, 38 subjects were included in the final analysis, 19 for each condition (tDCS and sham).

\section{Baseline Screening}

Groups showed no difference in age, sex, or years of education. Also, the neuropsychological tests did not reveal any differences between the two groups.

\section{Blood Pressure, Heart Rate, PANAS (Mood) Ratings}

No group differences were found in blood pressure, heart rate, and PANAS ratings at baseline and after the experimental session: No interactions [Time ( $1 \mathrm{hr}$ prior vs. before vs. after experimental session) $\times$ Group (tDCS vs. Sham)], or main effects for group were found for systolic and diastolic blood pressure, heart rate, and negative or positive PANAS ratings (all $p s>.1$ and all $p s>.05$, respectively). A main effect of time was found for heart rate $[F(2,17)=$ 12.93 and $p<.001$ ], with heart rate decreasing over time. No other main effects were found.

\section{Acquisition Task}

Participants in both groups were able to correctly remember sequences in this task, without significant differences between groups, as revealed by an unpaired $t$ test, with an average of $36.8(S D=15.47)$ correct recalls for the tDCS 
group and $30.8(S D=16.33)$ correct recalls for the sham group $[t(36)=1.42, p=.2]$. This indicates that tDCS over Broca's area did not significantly enhance working memory as such, although a trend in favor of the tDCS group emerged. Furthermore, there were no group differences in differential performance on strings of short and long lengths: We conducted a $2 \times 2$ ANOVA with the factor group (tDCS vs. sham) as a between-subjects factor and the factor length (short vs. long) as a within-subjects factor, where a half split of the data provided us with one sample of short strings (5-10 letters) and one of long strings (1112 letters). Only a main effect of string length was found $[F(1,36)=221.9, p<.001]$, with lower performance on long strings. There was no main effect of group $[F(1$, $36)=1.29, p=.26]$ or an interaction $[F(1,36)=0.008$, $p=.93]$. tDCS thus had no differential effect on the performance in the acquisition phase.

Because not all participants were equally fast in task performance, some participants completed the acquisition task within 20 min ( 8 in the sham group, and 5 in the tDCS group); hence, they received stimulation that covered the entire acquisition phase. For those participants who exceeded $20 \mathrm{~min}$, we analyzed the acquisition performance for the first $20 \mathrm{~min}$, and after $20 \mathrm{~min}$. A $2 \times 2$ ANOVA with group (tDCS vs. sham) and time (before vs. after 20 min) showed no significant interaction or main effects.

\section{Classification Task}

\section{Overall Analysis}

In the classification phase, where the participants had to classify novel strings, we found a significantly better overall performance for the tDCS group (72.1\% correct, $d^{\prime}=1.5$ ) as compared with the sham group $\left(66.4 \%\right.$ correct, $d^{\prime}=$
0.9), with $t(36)=2.13, p<.05$ for percentage correct and $t(36)=3.88, p<.001$ for $d^{\prime}$.

To further specify whether electrical stimulation on Broca's area affected the processing of grammaticality status and chunk strength information, or a combination of both, we first conducted a $2 \times 2 \times 2$ ANOVA with between-subjects factor group (tDCS vs. sham), and within-subjects factors grammaticality status (grammatical vs. nongrammatical items) and chunk strength (low vs. high), and with grammatical endorsement (percent perceived as grammatical) as the dependent variable. We found significant interactions for Group $\times$ Grammaticality status $[F(1,36)=4.57, p<.05]$, indicating that Broca's area is causally involved in the processing of grammaticality status. We also found a significant interaction for Chunk strength $\times$ Grammaticality status $[F(1,36)=15.1, p<$ $.001]$, indicating that both manipulations contributed to classification performance (see also Knowlton \& Squire, 1996, who reported a similar interaction). Note that the absence of a significant interaction Group $\times$ Chunk strength $[F(1,36)=0.74, p=.39]$ indicates endorsement rates were not affected by a combined effect of stimulation and chunk strength. In other words, Broca's area was not specifically related to the use of superficial cues to determine if an item was grammatical.

\section{Syntactic Violation Detection (Expressed in Endorsement Rates)}

A significant interaction of Group $\times$ Grammaticality, independent of chunk strength, was already found in the overall analysis. More specifically, the tDCS group was better at detecting syntactic violations (see Figure 3). As expected, the tDCS group had a significantly decreased false alarm rate, indicating a better ability to detect syntactic violation.
Figure 3. Significant interaction of Group $\times$ Grammaticality, collapsed over high and low chunk strength items, indicating that the tDCS group was significantly better to detect syntactic violations. Error bars denote SEM; * $p<.05$.

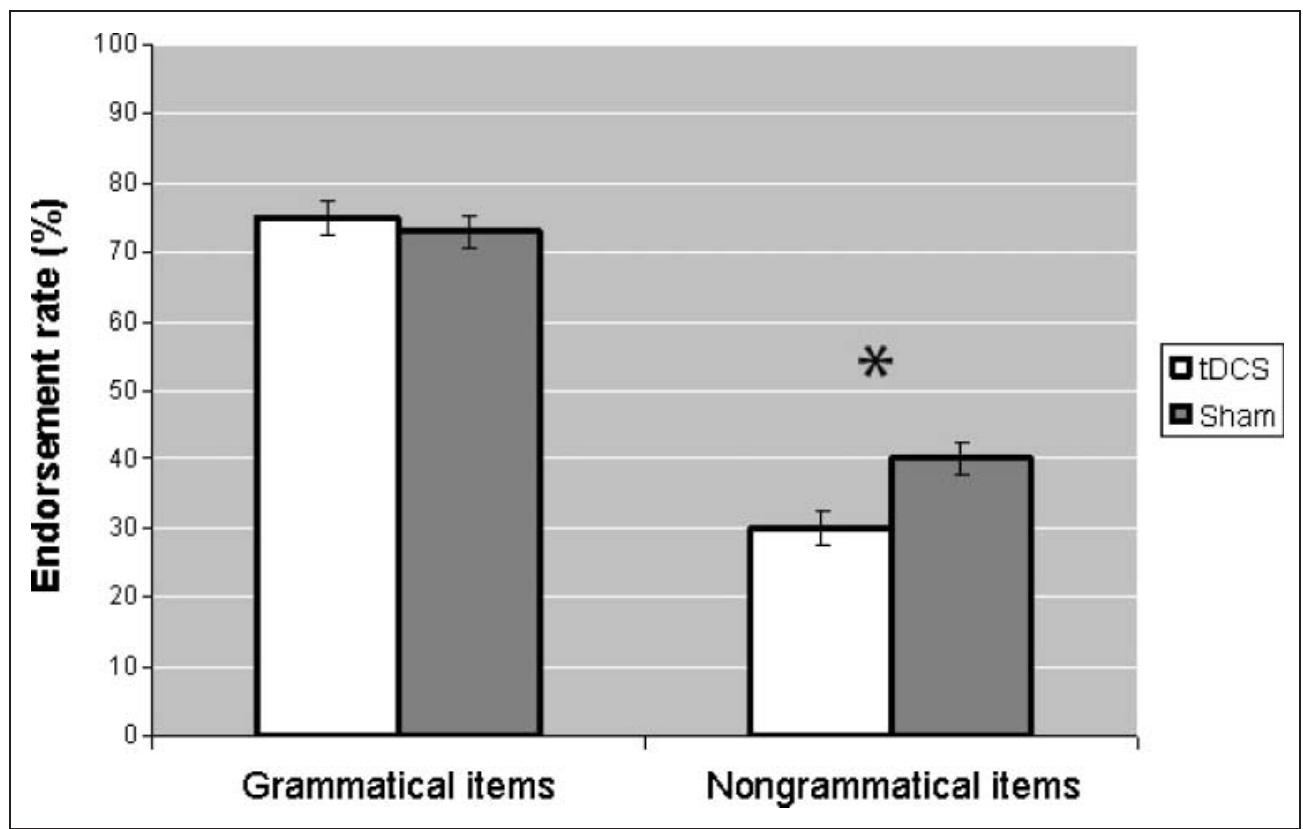


Figure 4. $d^{\prime}$ Values for Group $\times$ Chunk strength. The tDCS group was significantly better on the low chunk strength sample compared to sham. No such differences were found for the high chunk strength sample. Thus, the tDCS group performed better compared to sham on those items that did not provide superficial cues for classification (i.e., rule-based knowledge). Error bars denote SEM $; * p<.05$.

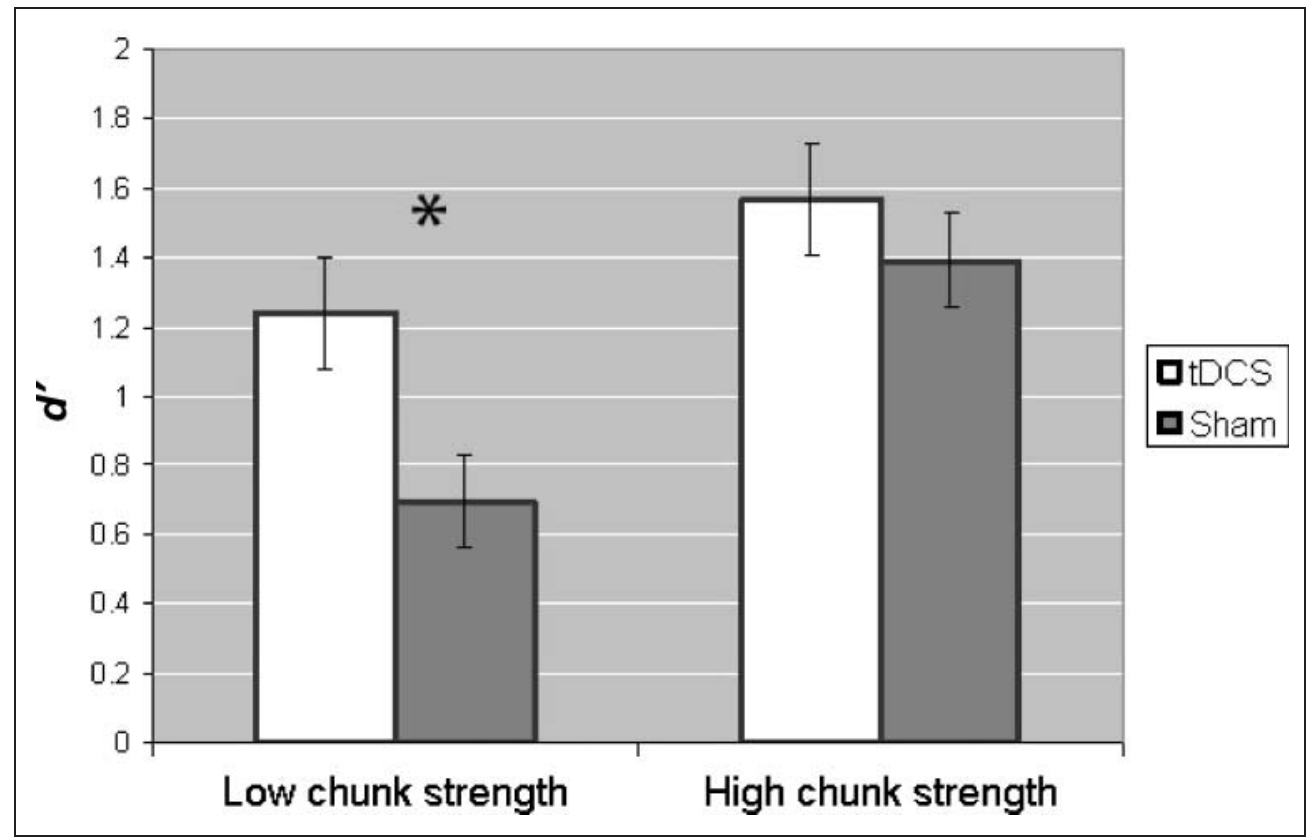

\section{Rule-based Knowledge (Expressed in $d^{\prime}$ )}

We analyzed if there was differential performance for the low chunk strength item sample compared to the high chunk strength item sample, while using $d^{\prime}$ as the dependent variable (i.e., discrimination accuracy). A $2 \times 2$ ANOVA with group (tDCS vs. sham) as the between-subjects factor and chunk strength (low vs. high) as the within-subjects factor did not reveal a significant interaction [but $p=.07$ with $F(1,36)=3.42$ ]. There was a main effect of chunk strength $[F(1,36)=27.37, p<.001]$, and post hoc $t$ tests showed that there was a significant group difference for performance on the low chunk strength items $\left[d^{\prime}=1.24\right.$ for the tDCS group and 0.69 for the sham group; $t(36)=$
$2.87, p<.01]$, but not for the high chunk strength items. These findings are similar to those found in Flöel et al. (2009), who found that white matter integrity around Broca's area correlated with the $d^{\prime}$ score on the low chunk strength items (see Figure 4).

\section{Rule-based Knowledge (Expressed in Endorsement Rate)}

To further analyze the differential performance for low and high chunk strength items, we now took into account grammaticality status; hence, we analyzed the endorsement rates for both samples separately (see Lieberman et al., 2004 and Knowlton \& Squire, 1996 for a similar analysis). A $2 \times 2$ ANOVA on the low chunk items revealed a
Figure 5. Endorsement rates for Group $\times$ Grammaticality, for both low and high chunk strength item samples. A significant interaction was found for low, but not for high, chunk strength items. The results indicate that the improved ability of the tDCS group to detect syntactic violations is specific to violations that bear low superficial resemblance. Error bars denote SEM; $* p<.05$.

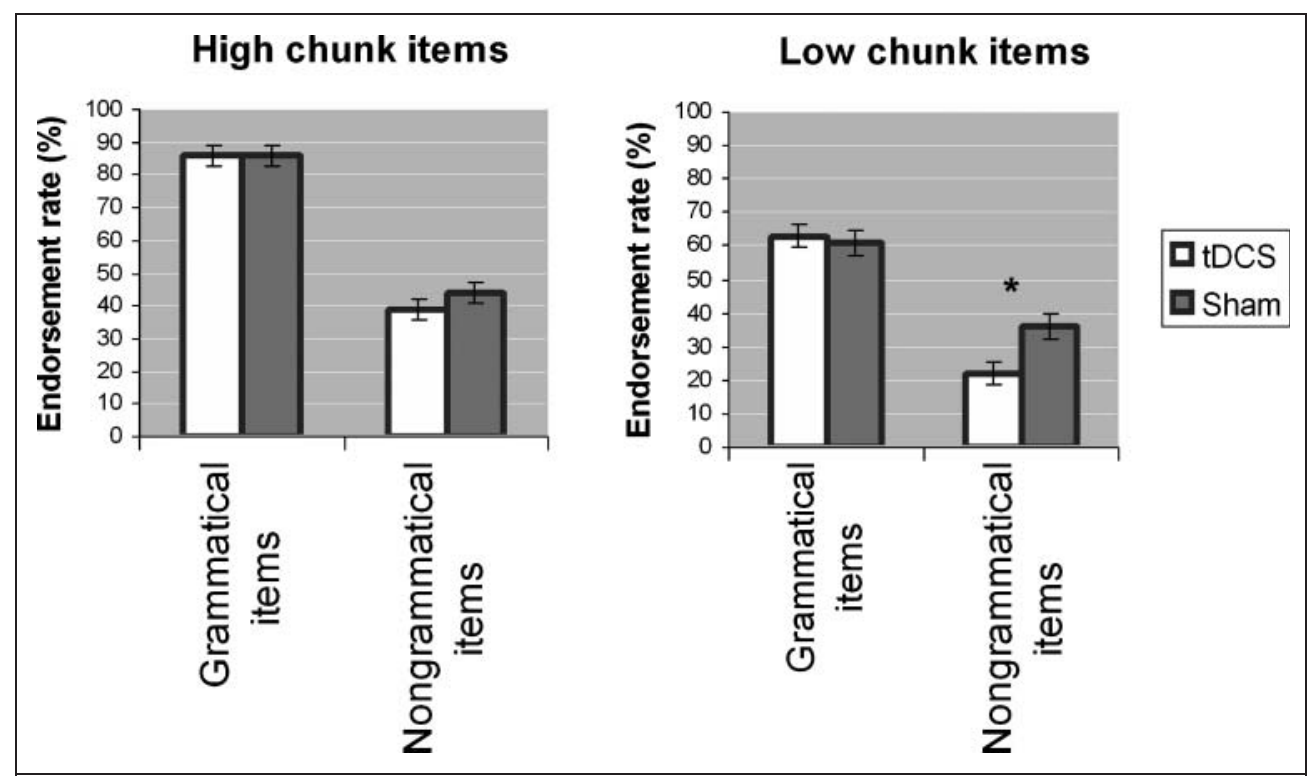


significant interaction between Group $\times$ Grammaticality status $[F(1,36)=7.23, p=.01]$, which was not the case for the high chunk items sample $[F(1,36)=1.01, p=.32]$ (Figure 5), indicating that the tDCS group was significantly better to discriminate grammatical from nongrammatical items; and specifically to detect nongrammatical items that bear low superficial familiarity to the training items.

We expected to find a lower false alarm rate for the tDCS group compared to the sham group, independent of chunk strength. This was indeed the case: We found a direct causal involvement of Broca's area for processing grammaticality status, given by the significant interaction Group $\times$ Grammaticality status. Indeed, this indicated a decreased false alarm rate for the tDCS group. We did not find a significant interaction for Group $\times$ Chunk strength, indicating that both groups processed superficial information in a similar way. Broca's area was, therefore, not specifically involved in superficial information processing.

We also expected to find a better performance on the low chunk strength items sample for the tDCS group compared to the sham group, expressed in $d^{\prime}$. Indeed, the tDCS group had a significantly better performance on the low chunk strength items than the sham group. This effect appeared to be driven by a significantly better ability to detect those syntactic violations that bear low superficial resemblance.

In summary, the analysis demonstrated that Broca's area is causally involved in the processing of grammaticality information, and not in superficial information, evidenced by a better ability to detect syntactic violations, and especially those that have low superficial resemblance to the training items.

\section{Post Hoc Control Group}

In order to investigate if the interaction between Group $\times$ Grammaticality that was found for the low chunk items was specific to anodal tDCS versus sham over the left prefrontal area, we conducted the same analysis and included the post hoc control group. The $3 \times 2$ ANOVA with the factors Group (tDCS Broca vs. tDCS Cz vs. Sham) $\times$ Grammaticality (Grammatical vs. Nongrammatical items), and the dependent variable, "endorsement rate," revealed a (marginally) significant interaction of Group $\times$ Grammaticality $[F(1,45)=2.77, p=.07]$. Post hoc $t$ test comparisons showed that syntactic violation detection of the additional control group (tDCS Cz) was similar to that of the sham group $[t(27)=0.93, p=.36]$, but (marginally) significantly different from the tDCS Broca group $[t(27)=$ $1.82, p=.07]$. No differences were found for the high chunk strength items sample.

\section{DISCUSSION}

\section{Main Findings}

In this study, we show for the first time that anodal tDCS over left BA 44/45 during the acquisition process of an arti- ficial grammar enhances subsequent classification performance. Enhanced performance was indicated by better performance on syntactic violation detection and rulebased decisions.

\section{Broca's Area and Processing of Natural Grammars}

Broca's area plays a major role in linguistic processing. A recent meta-analysis of functional neuroimaging studies of syntactic processing (Indefrey, 2001) concluded that the most reliably replicable finding independent of imaging techniques, presentation modes, and experimental procedures, was activation in BA 44/45, or the left inferior frontal gyrus. This is also consistent with what is known from brain lesion data (Caplan, 1992; Caramazza \& Zurif, 1976). The left inferior frontal gyrus is part of prefrontal cortex that has been implicated in different features of language processing, involving phonological, syntactic, semantic, pragmatic, as well as nonlinguistic contextual information (Petersson et al., 2004;Bookheimer, 2002; Mesulam, 2002; Duncan, 2001).

\section{Broca's Area and Processing of Artificial Grammars}

With regard to AGL tasks, Reber (1967) suggested that this type of implicit learning is intrinsic to natural language acquisition. Indeed, as already described briefly in the Introduction, Broca's area has repeatedly been found to be activated during the classification phase of the AGL paradigm in fMRI experiments (e.g., Forkstam et al., 2006; Petersson et al., 2004). The AGL paradigm has therefore been demonstrated to be a good model of natural language learning (Petersson et al., 2004). AGL tasks that differed from Reber's original task confirmed the activation in Broca's area (e.g., Bahlmann, Schubotz, \& Friederici, 2008; Friederici, Bahlmann, Heim, Schubotz, \& Anwander, 2006; Opitz \& Friederici, 2003). Furthermore, Broca's area was specifically linked to rule-based knowledge (Flöel et al., 2009; Forkstam et al., 2006; Opitz \& Friederici, 2004) and to syntactic violation detection (Udden et al., 2008; Forkstam et al., 2006; Petersson et al., 2004).

\section{Broca's Area and Detection of Syntactic Violations}

Our results indicate that anodal tDCS over Broca's area caused an improved ability to recognize syntactic violations. Broca's area has been implicated in violation detection repeatedly, both in natural (e.g., Indefrey, Hagoort, Herzog, Seitz, \& Brown, 2001; Embick, Marantz, Miyashita, O’Neil, \& Sakai, 2000; Ni et al., 2000; Kang, Constable, Gore, \& Avrutin, 1999) and in artificial (Udden et al., 2008; Forkstam et al., 2006; Petersson et al., 2004) language tasks.

As for natural language tasks, Indefrey et al. (2001) reported data on brain activations during language processing in an experiment where participants had to detect syntactic violations in meaningless sentences. The data revealed that syntactic error detection was specifically related 
to a region of left dorsolateral prefrontal cortex in or adjacent to Broca's area.

For AGL tasks, Udden et al. (2008) demonstrated in an r'TMS experiment that Broca's area was involved in syntactic violation detection. The same regional specificity was observed in fMRI experiments, where Broca's area was activated during detection of syntactic violations when classifying strings from an artificial grammar (Forkstam et al., 2006; Petersson et al., 2004). All these studies implemented a balanced chunk strength design. The novel insight from our study stems from the focus on the role of Broca's are in acquisition of an artificial grammar, which had not been investigated by previous studies: We showed that anodal tDCS on Broca's area during the acquisition of an artificial grammar affects subsequent classification of novel items, driven by an improved ability to detect syntactic violations.

\section{Broca's Area and Rule-based Learning}

We were also interested in the causal role of Broca's area in rule-based learning, as opposed to chunk-based learning. In previous studies, separate analysis on low versus high chunk strength items have implicated different brain areas for rule-based learning, as opposed to chunk-based learning, an important one being Broca's area (Flöel et al., 2009; Forkstam et al., 2006; Lieberman et al., 2004; Opitz \& Friederici, 2003, 2004). In our study, the tDCS group showed an improved classification performance on low chunk strength items (which show relatively low resemblance to the training items), as opposed to high chunk strength items. This is consistent with findings by Flöel et al. (2009), who showed that better performance on low chunk strength items is correlated with the integrity of white matter tracts arising from Broca's area. Thus, this study shows, for the first time, that Broca's area is causally involved in acquiring information of the rules underlying an (artificial) grammar.

\section{Grammaticality and Chunk Strength Information}

Our results demonstrate that the grammaticality effect on classification performance is dependent of superficial familiarity of the strings, irrespective of stimulation group. Several researchers have argued that the effect of grammaticality status might depend on relative chunk strength (e.g., Pothos, 2007; Perruchet \& Pacteau, 1990; Dulany, Carlson, \& Dewey, 1984). Classification of strings that contain bi- or trigrams that occurred frequently during the training phase might cause a feeling of familiarity, leading to the participant's perception of a particular string as grammatical (for an overview, see Udden et al., 2008). AGL studies that implemented a balanced chunk strength design have shown that chunk strength has some effect on endorsement rates (e.g., Forkstam et al., 2006; Meulemans \& Van der Linden, 1997; Knowlton \& Squire, 1996), but that this effect is smaller than the grammaticality effect. This was the case in our study, too, given that there was no significant interaction between stimulation group and chunk strength information on the endorsement rates, whereas there was such an interaction between group and grammaticality status.

There is no consistency in the literature whether the effects of grammaticality status and chunk strength information act independently of each other. Some studies show that this is, indeed, the case (Forkstam et al., 2006; Meulemans \& Van der Linden, 1997), but other studies find that these are dependent (Lieberman et al., 2004; Knowlton \& Squire, 1996). All these studies implemented a balanced chunk strength design.

\section{Limitations}

In the present study, unspecific effects of anodal tDCS were controlled for by the sham stimulation, and by anodal tDCS over a control site. Therefore, we feel confident that our results were, indeed, mediated by the well-described effects of anodal tDCS on learning systems. In future studies, right-hemispheric control sites should be included to further elucidate the role of right prefrontal cortex in grammar acquisition. Furthermore, we implemented a between-subjects design, which might have led to baseline differences between groups. However, age, sex, years of education, and neuropsychological performances were similar, indicating that the differential performance on grammaticality classification between the experimental groups were due to tDCS versus sham stimulation, and not to general group differences.

\section{Conclusions}

This study is the first to demonstrate that (1) stimulation of Broca's area during the learning phase influences subsequent performance on a grammatical classification task, indicating that Broca's area plays a causal role in the acquisition of an artificial grammar, and that (2) Broca's area is specifically involved in extracting grammatical rules, and especially in syntactic violation detection. Our findings support previous hypotheses from lesion (Caplan, 1992; Caramazza \& Zurif, 1976) as well as imaging (Forkstam et al., 2006; Petersson et al., 2004) data that Broca's area is specifically involved in the processing of grammaticality information, as opposed to superficial chunk strength information.

\section{Outlook}

The use of tDCS in our experiment is potentially relevant for further use in clinical settings. Persisting language deficits after a stroke contribute significantly to the quality of life in aphasic patients (Bayley et al., 2007). Our findings indicate that reacquisition of grammatical knowledge after brain lesions may be improved by tDCS administered during language training, a hypothesis that should be tested in future studies. 


\section{Acknowledgments}

This research was supported by an EU Sixth Framework Marie Curie Research Training Network Program on Language and Brain (www.hull.ac.uk/RTN-LAB/) to M. de V. and S. K., and the Deutsche Forschungsgemeinschaft [Fl 379-4/1] to A. F., the IZKF [Floe/3/004/08] to A. F., and the Bundesministerium für Forschung und Bildung [01GW0520] to A. F. We thank Anna Bastl for her help with data collection and the reviewers for their helpful comments.

Reprint requests should be sent to Agnes Flöel, Department of Neurology, Universitätsmedizin Charite, Chariteplatz 1, 10117 Berlin, Germany, or via e-mail: agnes.floeel@charite.de or Meinou H. de Vries, Max Planck Institute for Psycholinguistics, P.O. Box 310, 6500 AH Nijmegen, The Netherlands, or via e-mail: meinou. devries@mpi.nl.

\section{REFERENCES}

Bahlmann, J., Schubotz, R. I., \& Friederici, A. D. (2008). Hierarchical artificial grammar processing engages Broca's area. Neuroimage, 42, 525-534.

Bayley, M. T., Hurdowar, A., Teasell, R., Wood-Dauphinee, S., Korner-Bitensky, N., Richards, C. L., et al. (2007). Priorities for stroke rehabilitation and research: Results of a 2003 Canadian Stroke Network consensus conference. Archives of Physical Medicine and Rebabilitation, 88, 526-528.

Bookheimer, S. (2002). Functional MRI of language: New approaches to understanding the cortical organization of semantic processing. Annual Review of Neuroscience, 25, 151-188.

Caplan, D. (1992). Language: Structure, processing and disorders. Cambridge, MA: MIT Press.

Caramazza, A., \& Zurif, E. (1976). Dissociation of algorithmic and heuristic processes in language comprehension: Evidence from aphasia. Brain and Language, 3, 572-582.

Chomsky, N. (2000). New borizons in the study of language and mind. Cambridge, UK: Cambridge University Press.

Cleeremans, A., Destrebecqz, A., \& Boyer, M. (1998). Implicit learning: News from the front. Trends in Cognitive Sciences, 2, 406-416.

Dulany, D. E., Carlson, R. A., \& Dewey, G. I. (1984). A case of syntactical learning and judgment: How conscious and how abstract. Journal of Experimental Psychology: General, $113,541-555$.

Duncan, J. (2001). An adaptive coding model of neural function in prefrontal cortex. Nature Reviews Neuroscience, 2, 820-829.

Embick, D., Marantz, A., Miyashita, Y., O’Neil, W., \& Sakai, K. L. (2000). A syntactic specialization for Broca's area. Proceedings of the National Academy of Sciences, U.S.A., 97, 6150-6154.

Flöel, A., De Vries, M. H., Scholz, J., Breitenstein, C., \& Johansen-Berg, H. (2009). White matter integrity in the vicinity of Broca's area predicts grammar learning success. Neuroimage, 47, 1974-1981.

Flöel, A., Roesser, N., Michka, O., Knecht, S., \& Breitenstein, C. (2008). Noninvasive brain stimulation improves language learning. Journal of Cognitive Neuroscience, 20, 1415-1422.

Forkstam, C., Hagoort, P., Fernández, G., Ingvar, M., \& Petersson, K. M. (2006). Neural correlates of artificial syntactic structure classification. Neuroimage, 32, 956-967.

Friederici, A. D., Bahlmann, J., Heim, S., Schubotz, R. I., \& Anwander, A. (2006). The brain differentiates human and non-human grammars: Functional localization and structural connectivity. Proceedings of the National Academy of Sciences, U.S.A., 103, 2458-2463.
Hauser, M. D., Chomsky, N., \& Fitch, W. T. (2002). The faculty of language: What is it, who has it, and how did it evolve? Science, 298, 1569-1579.

Hochhaus, L. (1972). A table for the calculation of $\mathrm{d}^{\prime}$ and Beta. Psychological Bulletin, 77, 375-376.

Howard, D. V., \& Howard, J. H. (2001). When it does hurt to try: Adult age differences in the effect of instructions on implicit pattern learning. Psychonomic Bulletin \& Review, 8, 798-805.

Hummel, F., Celnik, P., Giraux, P., Flöel, A., Wu, W. H., Gerloff, C., et al. (2005). Effects of non-invasive cortical stimulation on skilled motor function in chronic stroke. Brain, 128, 490-499.

Indefrey, P. (2001). The neural architecture of syntactic parsing and encoding. Brain and Language, 79, 106-107.

Indefrey, P., Hagoort, P., Herzog, H., Seitz, R. J., \& Brown, C. M. (2001). Syntactic processing in left prefrontal cortex is independent of lexical meaning. Neuroimage, 14, 546-555.

Jackendoff, R. (2002). Foundations of language: Brain, meaning, grammar, evolution. Oxford, UK: Oxford University Press.

Kang, A. M., Constable, R. T., Gore, J. C., \& Avrutin, S. (1999). An event-related fMRI study of implicit phrase-level syntactic and semantic processing. Neuroimage, 10, 555-561.

Kincses, T. Z., Antal, A., Nitsche, M. A., Bartfai, O., \& Paulus, W. (2004). Facilitation of probabilistic classification learning by transcranial direct current stimulation of the prefrontal cortex in the human. Neuropsychologia, 42, 113-117.

Knowlton, B. J., \& Squire, L. R. (1996). Artificial grammar learning depends on implicit acquisition of both abstract and exemplar-specific information. Journal of Experimental Psychology: Learning, Memory, and Cognition, 22, 169-181.

Lieberman, M. D., Chang, G. Y., Chiao, J., Bookheimer, S. Y., \& Knowlton, B. J. (2004). An event-related fMRI study of artificial grammar learning in a balanced chunk strength design. Journal of Cognitive Neuroscience, 16, 427-438.

Liebetanz, D., Nitsche, M. A., Tergau, F., \& Paulus, W. (2002). Pharmacological approach to the mechanisms of transcranial DC-stimulation-induced after-effects of human motor cortex excitability. Brain, 125, 2238-2247.

Mesulam, M. M. (2002). The human frontal lobes: Transcending the default mode through contingent encoding. In R. T. Knight (Ed.), Principles of frontal lobe function (pp. 8-30). Oxford, UK: Oxford University Press.

Meulemans, T., \& Van der Linden, M. (1997). Associative chunk strength in artificial grammar learning. Journal of Experimental Psychology: Learning, Memory, and Cognition, 23, 1007-1028.

Ni, W., Constable, R. T., Mencl, W. E., Pugh, K. R., Fullbright, R. K., Shavwitz, S. E., et al. (2000). An event-related neuroimaging study distinguishing form and content in sentence processing. Journal of Cognitive Neuroscience, 12, 120-133.

Nitsche, M. A., Doemkes, S., Karaköse, T., Antal, A., Liebetanz, D., Lang, N., et al. (2007). Shaping the effects of transcranial direct current stimulation of the human motor cortex. Journal of Neurophysiology, 97, 3109-3117.

Nitsche, M. A., Liebetanz, D., Lang, N., Antal, A., Tergau, F., \& Paulus, W. (2003). Criteria for transcranial direct current stimulation (tDCS) in humans. Clinical Neurophysiology, 114, 2220-2222.

Nitsche, M. A., Schauenburg, A., Lang, N., Liebetanz, D., Exner, C., Paulus, W., et al. (2003). Facilitation of implicit motor learning by weak transcranial direct current stimulation of the primary motor cortex in the human. Journal of Cognitive Neuroscience, 15, 619-626. 
Opitz, B., \& Friederici, A. (2003). Interactions of the hippocampal system and the prefrontal cortex in learning language-like rules. Neuroimage, 19, 1730-1737.

Opitz, B., \& Friederici, A. (2004). Brain correlates of language learning: The neuronal dissociation of rule-based versus similarity-based learning. Journal of Neuroscience, 24, 8436-8440.

Perruchet, P., \& Pacteau, C. (1990). Synthetic grammar learning: Implicit rule abstraction of explicit fragmentary knowledge. Journal of Experimental Psychology: General, 199, 264-275.

Petersson, K. M., Forkstam, C., \& Ingvar, M. (2004). Artificial syntactic violations activate Broca's region. Cognitive Science, 28, 383-407.

Pothos, E. M. (2007). Theories of artificial grammar learning. Psychological Bulletin, 133, 227-244.

Purpura, D. P., \& McMurtry, J. G. (1965). Intracellular activities and evoked potential changes during polarization of motor cortex. Journal of Neurophysiology, 28, 166-185.

Reber, A. S. (1967). Implicit learning of artificial grammars. Journal of Verbal Learning \& Verbal Behavior, 6, 855-863

Reis, J., Schambra, H. M., Cohen, L. G., Buch, E. R., Fritsch, B., Zarahn, E., et al. (2009). Noninvasive cortical stimulation enhances motor skill acquisition over multiple days through an effect on consolidation. Proceedings of the National Academy of Sciences, U.S.A., 106, 1590-1595.

Udden, J., Folia, V., Forkstam, C., Ingvar, M., Fernandez, G., Overeem, S., et al. (2008). The inferior frontal cortex in artificial syntax processing: An rTMS study. Brain Research, 1224, 69-78.

Watson, D., Clark, L. A., \& Tellegen, A. (1988). Development and validation of brief measures of positive and negative affect: The PANAS Scales. Journal of Personality and Social Psychology, 47, 1063-1070. 